\title{
Analysis of the properties of silicone rubber maxillofacial prosthetic materials
}

\author{
Tariq Aziz ${ }^{a}$, Mark Waters ${ }^{a, *}$, Robert Jagger ${ }^{b}$
}

\author{
a Departments of Basic Dental Science, University of Wales College of Medicine, Heath Park, Cardiff, UK \\ ${ }^{\mathrm{b}}$ Adult Dental Health, University of Wales College of Medicine, Heath Park, Cardiff, UK
}

Accepted 28 October 2002

\author{
KEYWORDS \\ Silicone rubber; \\ Maxillofacial; Prosthesis; \\ Mechanical properties
}

\begin{abstract}
Summary Objectives. Maxillofacial prosthetic materials are used to replace facial parts lost through disease or trauma. Silicone rubbers are the materials of choice, however it is widely accepted that these materials do not possess ideal properties. The objective of this study was to assess the properties of a range of commercially available silicone rubber maxillofacial materials and make recommendations for improvements.

Methods. Specimens of five commonly used maxillofacial materials were prepared in dental flasks according manufacturers instructions. Tear strength, tensile strength, percentage elongation, hardness, water absorption and water contact angles were determined for each material.

Results. The tear strength of Factor II, Cosmesil HC and Nusil were all comparable and significantly higher than Cosmesil St and Prestige $(p<0.001)$. Nusil had a significantly higher tensile strength and elongation in comparison to the other materials $(p<0.001)$ and Cosmesil St and Cosmesil HC were significantly harder $(p<0.001)$. Factor II was significantly less wetted and Prestige and Cosmsesil St had a significantly higher water absorption in comparison to the other materials.

Conclusions. None of the commercially available silicone rubber materials possessed ideal properties for use as a maxillofacial prosthetic material. Factor II, however, showed more favourable properties due to it's high tear strength, softness and ease of manipulation.

(c) 2003 Elsevier Science Ltd. All rights reserved.
\end{abstract}

\section{Introduction}

Maxillofacial materials are used to replace missing facial parts which have been lost through disease or trauma. They are usually comprised of poly(dimethylsiloxane) (PDMS) elastomers. Although widely used these materials are far from ideal.

*Corresponding author. Tel.: +44-2920-742610; fax: +442920-744509.

E-mail address: watersmg@cf.ac.uk
The quality of these materials depends greatly on their two basic components, the PDMS chains and the silica fillers, and the interactions between these two components affects the overall strength and service life of the material. The following physical properties are essential in a material used for the construction of maxillofacial prostheses: ${ }^{[1-4]}$

1. Clinically the most important physical property is the tear strength of the material. The tear strength of a PDMS maxillofacial material is 
extremely important particularly at the thin margins surrounding nasal and eye prostheses. This thin margin helps to mask the presence of a facial prosthesis to the surrounding facial tissue. The thin margins of the prosthesis is usually glued with medical adhesive to the patients face. When the facial prosthesis is removed, usually at night time or for cleaning, the thin margins are susceptible to tearing as the prosthesis is gently peeled away from the facial tissue. The facial prosthesis is then permanently damaged and has to be replaced. Therefore it is important that a material with a high resistance to tearing is used to construct these prostheses.

2. The tensile strength of the silicone elastomer gives an overall strength of the material and the resulting elongation gives an indication of the flexibility of the prosthesis. A prosthesis with a high elongation at break is desirable especially when peeling a nasal or eye prostheses from facial tissue.

3. The hardness of the maxillofacial material is also a measure of flexibility and is important since it is desirable to have a material with similar hardness to the missing facial tissue.

4. The water absorption of the prosthetic material is important since facial prostheses may absorb saliva or sweat from surrounding facial tissue, and also after washing the prosthesis in water. Any absorbed water may affect the physical properties and also affect the perception of colour matching to the surrounding facial tissue. ${ }^{[5]}$

5. Poor surface wettability by saliva leads to poor boundary lubrication and thus patient discomfort. ${ }^{[6,7]}$ This is due to a low surface energy of the facial materials producing a high contact angle with water and therefore preventing the spreading of water over the surface. ${ }^{[8-10]}$

All these properties must be taken into account when producing a novel maxillofacial prosthetic material. In addition it is important that the material is easy to manipulate and process to make it accepted by maxillofacial technicians.

Previous studies have compared silicone maxillofacial materials, ${ }^{[11-13]}$ however, there is little information regarding the newer materials which are available today. Previous studies have revealed a wide variety of testing methods and conditions used to examine the mechanical and physical properties of poly(dimethylsiloxane) maxillofacial materials and therefore it is difficult to compare one study with another. For example, the tear resistance of maxillofacial materials is a complex phenomena and dependent on the shape of the test specimens and also on the speed of testing (crosshead speed). The higher the rate of stressing the less time the molecules have to redistribute the stress and this leads premature tearing of the specimen thus indicating a low value for tear strength. Therefore data on tear strength of maxillofacial materials has to be carefully interpreted. All studies agree that further improvements in the mechanical and physical properties are necessary for poly(dimethylsiloxane) maxillofacial materials in particular the thin margin tear strength, elasticity and hardness.

Therefore the main aim of this study was to analyse a range of clinically important properties for the commercially available maxillofacial materials in order to help in the design of a new maxillofacial prosthetic material:

\section{Materials and methods}

The commercial materials used and the curing conditions employed for each material in this study are shown in Table 1.

\section{Hardness test}

Hardness specimens $(45 \mathrm{~mm} \times 45 \mathrm{~mm} \times 4 \mathrm{~mm})$ were made using the conventional dental flasking technique. Five hardness specimens were prepared for each material.

The hardness test used in this study was based on measurements of the indentation of a rigid ball into the test specimen under specified conditions and conducted according to I.S.O /R.48 (1968) (E); A.S.T.M. D-1415 (1983). BS 903 PT A. 26 (1969);

Table 1 Curing conditions for commercial materials.

\begin{tabular}{llll}
\hline Materials & Curing system & Curing conditions & Manufacturer \\
\hline Cosmesil high compliance (HC) & 3 Part condensation & $24 \mathrm{~h}$ at $100{ }^{\circ} \mathrm{C}$ & Principality Medical, Newport, UK \\
Cosmesil standard (St) & 3 Part condensation & $24 \mathrm{~h}$ at $100^{\circ} \mathrm{C}$ & Principality Medical, Newport, UK \\
A-2186 (Factor II) & 2 Part addition & $1 \mathrm{~h}$ at $100{ }^{\circ} \mathrm{C}$ & Factor II, Lakeside, CA, USA \\
Premium facial and body elastomer (Prestige) & 2 Part addition & $1.5 \mathrm{~h}$ at $100^{\circ} \mathrm{C}$ & Prestige Dental, Bradford, UK \\
MED-4920 (Nusil) & 2 Part addition & $3 \mathrm{~h}$ at $120^{\circ} \mathrm{C}$ & Nusil Technology, Carpineteria, CA, USA \\
\hline
\end{tabular}


using a Wallace Dead Load Hardness Tester (H.W. Wallace and CO Ltd, Croydon, UK) with results being read directly in international rubber hardness degrees (IRHD). For each specimen, 10 hardness readings were measured at 10 different positions on the surface of the specimen.

\section{Tensile test}

Tensile specimens $(100 \mathrm{~mm} \times 80 \mathrm{~mm}$ and $2 \mathrm{~mm})$ were made using the conventional dental flasking technique. Ten dumb-bell shaped specimens (Fig. 1) of rubber material (in accordance with BS 903 (1979) type 2 test pieces) were cut from strips of processed material.

The specimens were tested according to BS 903 Part A2 (1979) determination of tensile stress strain properties. The test was carried out on Lloyd Instruments LR10K tensile machine fitted with a $1 \mathrm{kN}$ load cell linked to an IBM compatible computer (Lloyd Instruments Ltd, Fareham, Hampshire, UK).

Tensile strength and percentage elongation were calculated automatically by the software using the equations below

Stress $\left(\mathrm{Nm}^{-2}\right)=\frac{\text { Load }}{\text { Initial cross-sectional area }}$

Percentage Strain $(\%)=\frac{\text { Extension }}{\text { Original length }} \times 100$

\section{Tear test}

Test specimens $(100 \mathrm{~mm} \times 80 \mathrm{~mm}$ and $2 \mathrm{~mm})$ were made using the conventional dental flasking

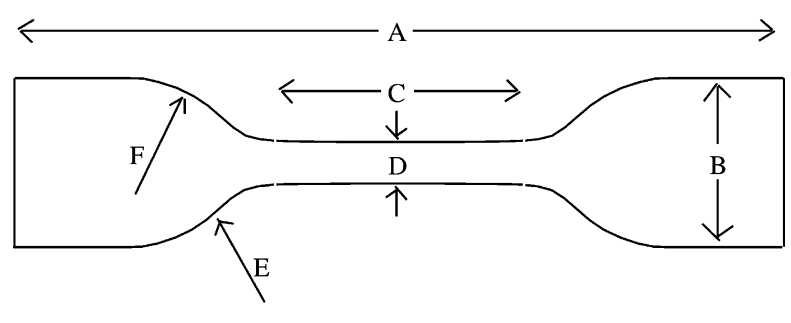

Dimensions Type $2(\mathrm{~mm})$

$\begin{array}{ll}\text { A } & 75 \text { minimum } \\ \text { B } & 12.5 \pm 1 \\ \text { C } & 25 \pm 1 \\ \text { D } & 4 \pm 0.1 \\ \text { E } & 8 \pm 0.5 \\ \text { F } & 12.5 \pm 1.0\end{array}$

Figure 1 Dimensions of dumb-bell tensile test specimen. technique. Ten tear specimens were cut from the processed material with final dimensions of $50 \mathrm{~mm} \times 10 \mathrm{~mm} \times 2 \mathrm{~mm}$, with a $4 \mathrm{~mm}$ cut placed from one edge. This test specimen is a modification of the test specimen described by ASTM D624: standard test method for rubber property-tear resistance.

Testing was carried out using the Lloyd Instruments LR $10 \mathrm{~K}$ testing machine fitted with a $1 \mathrm{kN}$ load cell. Specimens were tested at a constant crosshead speed of $20 \mathrm{~mm} / \mathrm{min}$ at a gauge length of $25 \mathrm{~mm}$. On failure of the specimen the computer software automatically calculated the tear resistance by using the following equation

$\mathrm{Ts}=F / t$

where: Ts the tear resistance $(\mathrm{N} / \mathrm{mm}) ; F$ the load at failure $(\mathrm{N})$; $t$ the thickness of specimen $(\mathrm{mm})$.

\section{Water absorption}

Water absorption test specimens $(45 \mathrm{~mm}$ in diameter and $1 \mathrm{~mm}$ thick) were made using the conventional dental flasking technique. Five test specimens were constructed for each material.

The specimens were placed in a desiccator containing phosphorus pentoxide and calcium chloride until they achieved a constant weight to an accuracy of $0.0001 \mathrm{~g}$. The specimens were then placed in glass screw topped jars containing distilled water and maintained in an oven at $37^{\circ} \mathrm{C}$ and was changed weekly. At recorded intervals the specimens were removed, blotted to remove excess water and re-weighed, again to an accuracy of $0.0001 \mathrm{~g}$. The increase in mass was measured monthly for a period of 6 months for each of the five specimens. Percentage weight change was calculated by using the following equation

$\%$ Absorption $=\frac{W_{2}-W_{3}}{W_{1}}$

where $W_{1}$ the initial weight; $W_{2}$ the weight after absorption of water; $W_{3}$ the weight after desiccation.

\section{Dynamic contact angle analysis}

PDMS rubber rectangular specimens $(20 \mathrm{~mm} \times 10$ $\mathrm{mm} \times 1 \mathrm{~mm}$ ) were constructed using the standard dental flasking. Five test specimens were made for each material.

Care was taken not to handle the surfaces of the specimens in order to reduce the chance of contamination. Testing was carried out using a Cahn Dynamic Contact Angle Analyser model 312 (Cahn Instruments, Inc. Cerritos, California, USA) 


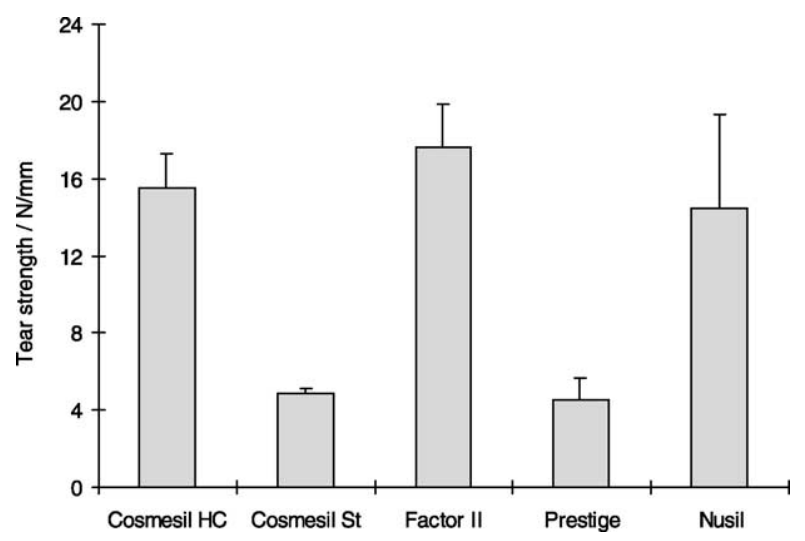

Figure 2 Tear strength of the commercially available materials.

linked to a Dan 386 IBM compatible computer (Dan Technology plc, London). The perimeter of the samples were measured individually for each specimen and analysed using the computer software. Care was taken so that each sample entered the wetting medium parallel to the surface. The specimens entered the wetting medium of distilled water at a speed of $30 \mu \mathrm{m} / \mathrm{s}$. The meniscus at the specimen water interface is characterised by the dynamic contact angle. A graphical display was shown on the computer as the experiment progressed, showing the wetting force of the sample as measured by the microbalance as it moves first into (lower line) and then out of (upper line) the water. This enabled the advancing contact angle to be calculated by least squares analysis of the bottom line and the receding contact angle to be calculated by least squares analysis of the top line using equation below

$\cos \theta=\frac{F}{p v}$

where $F$ the force $(\mathrm{mN}), p$ the perimeter $(\mathrm{m}), v$ the surface tension $(\mathrm{mN} / \mathrm{m}), \theta$ the contact angle.

\section{Statistics}

One way analysis of variance (ANOVA) was used to test for any significant difference between the mean values of the materials tested. Post-tests
(Bonferroni method) were used to determine whether the mean value of any particular material differed significantly from another specified material, while considering all the data.

\section{Results}

The tear strengths of the commercially available materials are shown in Fig. 2 and Table 2. Overall there were significant differences in the mean tear strengths between the commercially available materials (ANOVA). Post-tests (Bonferroni method) showed there was no significant difference $(p>0.05)$ in the tear strength of Factor II, Cosmesil $\mathrm{HC}$ and Nusil all three having significantly $(p<0.001)$ greater tear strength than Cosmesil St and Prestige.

The tensile strength of the commercially available materials is shown in Fig. 3 and Table 2. Nusil had a significantly higher tensile strength in comparison to the other materials $(p<0.001)$. There was no significant difference in the tensile strength of Cosmesil HC, Cosmesil St and Factor II. The tensile strength of Prestige was significantly lower than all the other commercially available materials $(p<0.001)$.

The elongation at break for the commercially available materials is shown in Table 2 and Fig. 4. Nusil had a significantly greater elongation at break in comparison to the other materials $(p<0.001)$. The elongation at break of Cosmesil HC was significantly greater than Cosmesil St, Prestige and Factor II $(p<0.001)$ but there was no significant difference $(p>0.05)$ in the elongation at break of Cosmesil St, Prestige and Factor II.

The hardness of the commercially available materials is shown in Table 2 and Fig. 5. There was no significant difference $(p>0.05)$ in the hardness of Cosmesil St and Cosmesil HC and both were significantly harder $(p<0.001)$ than Factor II, Prestige and Nusil.

The contact angle values for the commercially available materials are shown in Table 3. Post-tests (Bonferroni method) show that there were no significant differences between the advancing

Table 2 Properties of commercial maxillofacial materials.

\begin{tabular}{|c|c|c|c|c|c|}
\hline & Cosmesil & Cosmesil HC & Factor II & Prestige & Nusil \\
\hline Tear $(\mathrm{N} / \mathrm{mm})$ & $4.87 \pm 0.25$ & $15.55 \pm 1.77$ & $17.63 \pm 2.21$ & $4.53 \pm 1.1$ & $14.45 \pm 4.91$ \\
\hline Tensile $\left(\mathrm{N} / \mathrm{mm}^{2}\right)$ & $4.24 \pm 0.48$ & $3.87 \pm 0.33$ & $4.23 \pm 0.39$ & $2.53 \pm 0.19$ & $8.36 \pm 0.97$ \\
\hline Elongation (\%) & $577.1 \pm 58.1$ & $888 \pm 57.6$ & $650.8 \pm 41.1$ & $725.4 \pm 52$ & $1699 \pm 61.4$ \\
\hline Hardness (I.R.H.D) & $44.99 \pm 0.91$ & $44.47 \pm 0.36$ & $16.26 \pm 2.53$ & $16.21 \pm 0.43$ & $24.33 \pm 0.76$ \\
\hline
\end{tabular}




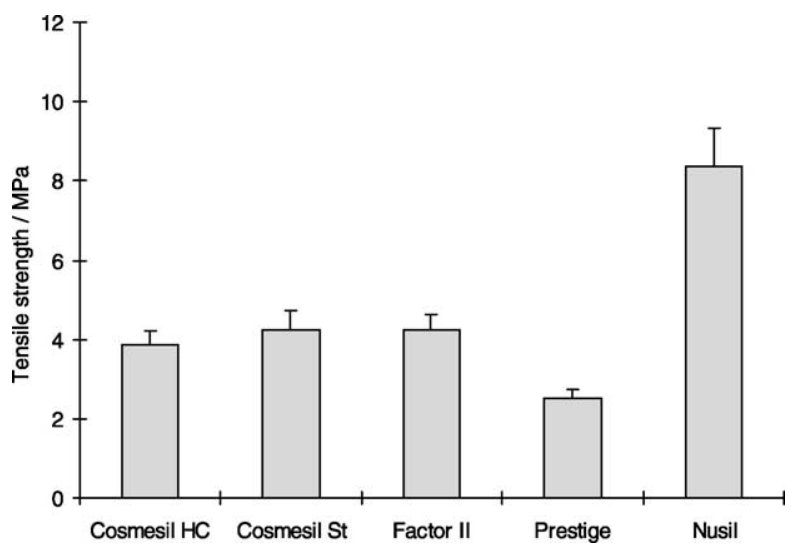

Figure 3 Tensile strength of the commercially available materials.

contact angle readings for all the commercially available materials $(p>0.05)$, except for Factor II which had a significantly higher advancing contact angle in comparison to Cosmesil $\mathrm{HC}$ and Cosmesil St $(p<0.05)$. There was no significant difference $(p>0.05)$ in the receding contact of Cosmesil HC and Cosmesil St both results being significantly lower than Factor II $(p<0.01)$, Prestige $(p<0.05)$ and Nusil $(p<0.01)$. There was no significant difference in the receding angle readings for Factor II, Prestige and Nusil $(p>0.05)$. There was no significant difference $(p>0.05)$ between the receding contact angles for Factor II, Prestige and Nusil which were significantly greater than the receding contact angles for Cosmesil $\mathrm{HC}$ and Cosmesil St. There was no significant difference in the mean equilibrium contact angle readings for all the materials tested $(p>0.05)$.

The water uptake for Cosmesil St and Prestige was much higher than that of the other materials so these materials are represented in different figures.

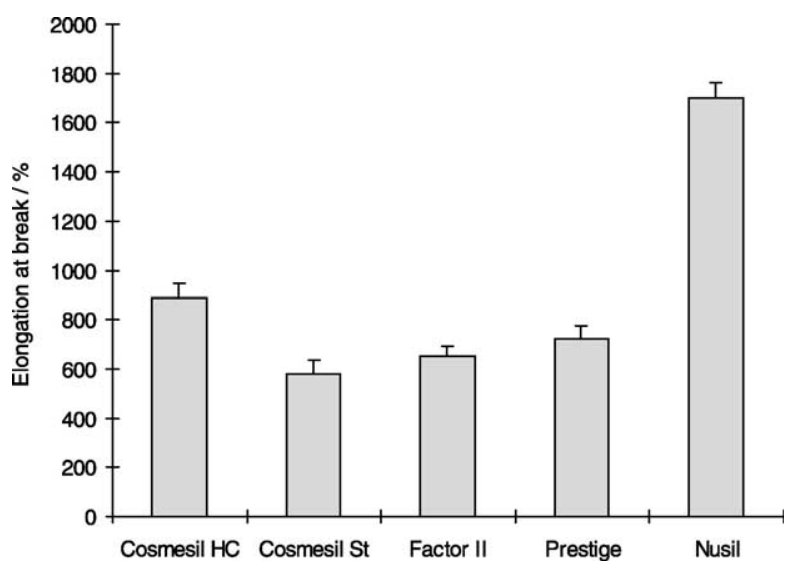

Figure 4 Elongation at break of the commercially available materials.

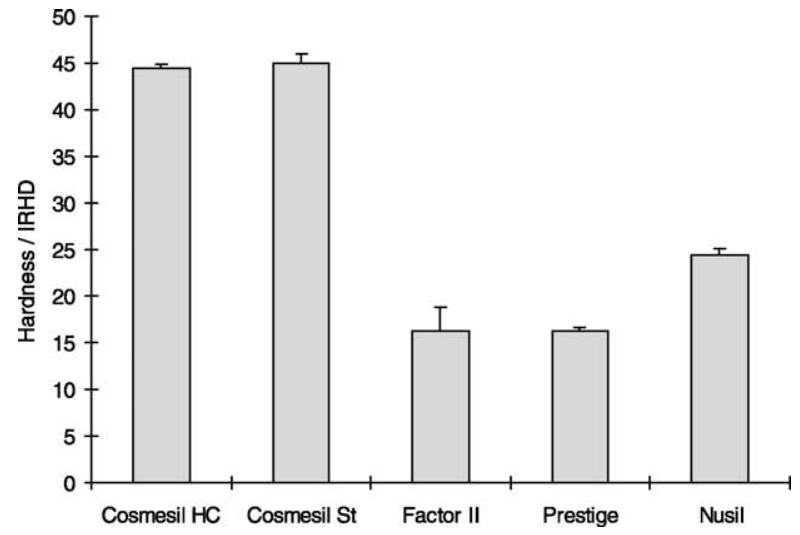

Figure 5 Hardness of the commercially available materials.

Following a period of 12 months in water, Cosmesil St $(33.98 \pm 0.43 \%)$ had a significantly higher level of water absorption when compared to Prestige (26.15 $\pm 1.78 \%$ ) (Fig. 6). Cosmesil St and Prestige had significantly greater water absorption when compared to the other materials $(p<0.001)$. There was no significant difference in water uptake of Cosmesil HC $(1.01 \pm 0.12 \%)$, Factor II $(3.46 \pm 0.82 \%)$ and Nusil $(1.80 \pm 1.41 \%)$ as shown in Table 4.

\section{Discussion}

The desirable properties of a material used as a maxillofacial prosthetic material include high tear strength, tensile strength and elongation at break, a low hardness and water absorption and good surface wettability. The results suggest that none of the tested commercial materials fulfilled the above criteria.

The differences observed in the physical and mechanical properties of the commercial materials are due to different components used in their formulations. More specifically the variables may include different cross-linking systems (addition or

\begin{tabular}{|c|c|c|c|}
\hline Tests (mean \pm SD) & $\begin{array}{l}\text { Advancing } \\
\text { angle } / \theta_{\text {Add }}\end{array}$ & $\begin{array}{l}\text { Receding } \\
\text { angle } / \theta_{\mathrm{Rec}}\end{array}$ & $\begin{array}{l}\text { Equilibrium } \\
\text { angle } / \theta_{\text {Equ }}\end{array}$ \\
\hline Cosmesil HC & $90.8 \pm 10.6$ & $26.19 \pm 6.4$ & $67.6 \pm 3.1$ \\
\hline Cosmesil St & $92.0 \pm 5.4$ & $29.24 \pm 2.7$ & $68.9 \pm 2.7$ \\
\hline Factor II & $114.3 \pm 9.1$ & $40.76 \pm 2.0$ & $74.1 \pm 3.9$ \\
\hline Prestige & $100.6 \pm 5.2$ & $38.42 \pm 4.7$ & $72.3 \pm 1.6$ \\
\hline Nusil & $103.1 \pm 4.7$ & $44.0 \pm 4.3$ & $72.9 \pm 2.9$ \\
\hline
\end{tabular}




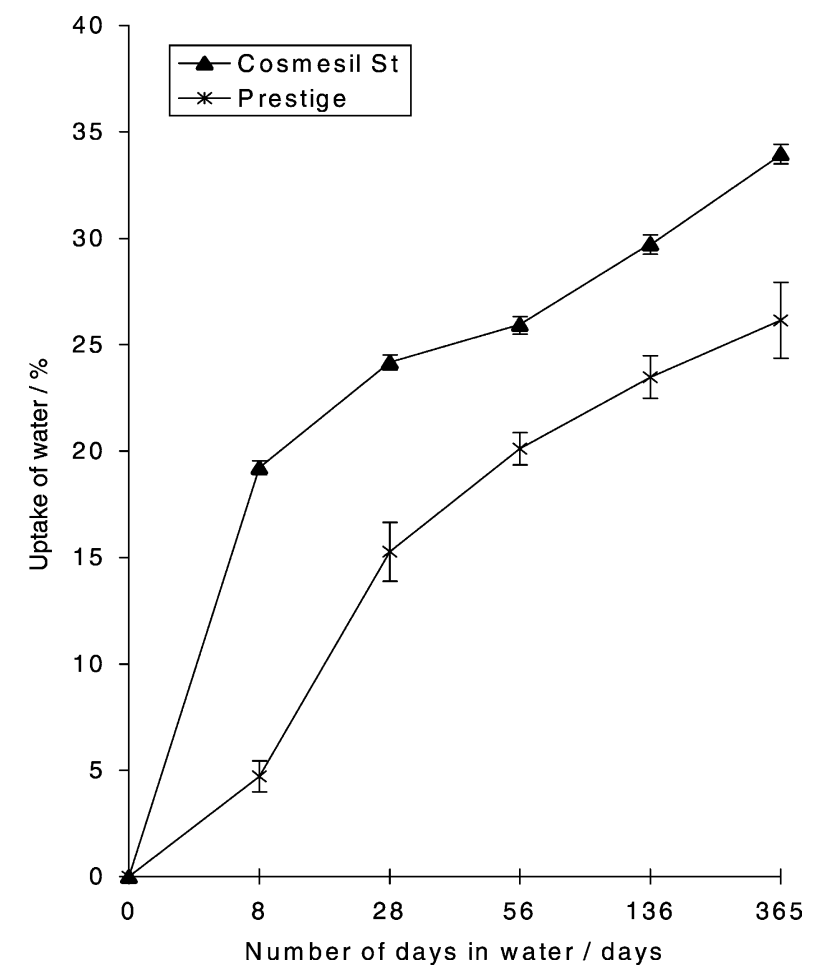

Figure 6 Water absorption for Cosmesil St and Prestige.

condensation), differences in the molecular weight of the PDMS, differences in the cross-link density and also differences in the grade and concentration of the silica filler used in the systems.

The commercial materials with the poorest tear and tensile properties were Prestige and Cosmesil St and these are also likely to contain non-surface treated fillers with a large surface area irrespective of the molecular weight of the polymer used in these systems. The interaction between the $-\mathrm{OH}$ groups on the filler and PDMS chains in these materials is not strong enough to prevent the material from rupturing under an applied force. As evidence of this it was noted that there was smooth tearing in the direction of the cut in the tear samples in these materials. In general, cross-linking of a low molecular weight PDMS produces an inelastic plastic material and results in rupture at very low deformations as experienced with Prestige.
In contrast Cosmesil HC, Nusil (MED-4920) and Factor II (A-2186) produced superior tensile strengths, tear strengths and elongation at break in comparison to the other commercial materials. It is likely these materials are composed of a high molecular weight PDMS chains in combination with surface treated silica filler. Cross-linking of a high molecular weight polymer produces a highly elastic material but also increases the viscosity of the resulting base polymer. The base viscosity for Cosmesil HC, Cosmesil St and Nusil was significantly greater than the base viscosity of Factor II and Prestige. The end-linking of low or medium molecular weight PDMS chains in combination with the addition cross-linking the reaction also increases the length of PDMS chains and produces a very elastic cross-linked network and this may account for the relatively low viscosity and very high mechanical properties observed for Factor II.

The interaction between the silica filler and the polymer chains influences the mechanical strength of the polymer matrix of the commercial materials. A silica filler with a high surface area maximises the polymer/filler interactions. If the silica filler particles used in the construction of the commercial are surface modified with dimethyl silyl or trimethyl silyl groups, the resulting polymer matrix is able to withstand greater deformation without rupture or tearing. The surface modification allows the PDMS chains to slip over the silica particles. Effectively, a more flexible network is produced and the previously disordered tangle of polymer chains are now able to be stretched out lengthways and a higher number of hydrogen bridge linkages act in the direction of the force, sufficiently high to induce crystallisation and hence the mechanical strength is increased. The surface groups on these silica particles repel water molecules and hence prevent water absorption into the cured material. As evidence of this it was noted that there was significant resistance to tearing in samples constructed from Cosmesil HC, Nusil nad Factor II. The tearing characteristics may be described as 'stickslip' tearing in which the material would initially tear then resist tearing for a time and then continue tearing to break. The tear path in these materials was in the direction of the applied force, effectively

Table 4 Water absorption for Cosmesil HC, Factor II and Nusil.

\begin{tabular}{|c|c|c|c|c|c|}
\hline Time in water (days) & 8 & 28 & 56 & 136 & 365 \\
\hline Cosmesil HC & $0.9 \pm 0.16$ & $1.13 \pm 0.20$ & $1.17 \pm 0.16$ & $1.10 \pm 0.13$ & $1.01 \pm 0.12$ \\
\hline Factor II & $1.4 \pm 0.56$ & $2.50 \pm 0.54$ & $3.00 \pm 0.57$ & $3.30 \pm 0.71$ & $3.46 \pm 0.82$ \\
\hline Nusil & $0.98 \pm 0.16$ & $1.65 \pm 0.78$ & $1.74 \pm 1.06$ & $1.60 \pm 0.80$ & $1.80 \pm 1.41$ \\
\hline
\end{tabular}


at a right angle to the direction of the cut in placed in the tear sample. The commercial material with the highest elongation at break was Nusil but the material with the greatest tear strength was Factor II. This may be due to Nusil being composed of only very high molecular weight PDMS chains and therefore producing the greatest degree of crystallisation when elongated but experiencing a lower resistance to tearing due to the greater distance between cross-links. The lower tensile strength and elongation at break for Cosmesil HC in comparison to Nusil may due to a slightly lower molecular weight of the PDMS but this reduction in tensile properties is outweighed by the increase in the tear strength due to the reduced distance between the cross-links. The highest tear strength was achieved by Factor II and this may be due to a broader bimodal PDMS molecular weight distribution. This may increase the tear strength of the cross-linked network by creating a local high cross-link density between the relatively long end-linked PDMS chains. The extra cross-links from the lower molecular weight polymer tighten the cross-linked network while at the same time retaining the flexibility of the long PDMS polymer chains and therefore increases the resistance to tearing. ${ }^{[14]}$

The hardness of the commercial materials ranged from 16 to $45 \mathrm{IRHD}$. The differences in the hardness readings may be due to differences in the cross-linking system, cross-link density, molecular weight of the polymer and differences in the grade and concentration of the silica filler. The condensation curing materials Cosmesil St and Cosmesil HC were the hardest materials. Both of these materials are likely to have contained high molecular weight polymers in a highly cross-linked network in combination with a very high filler concentration. A material with a very high cross-link density produces a very dense and hard material but also a very high filler concentration increases polymer/filler interactions and therefore reduces the mobility of the polymer chains. The addition curing materials, Nusil, Prestige and Factor II produced softer elastomers. This may be due to a lower silica filler concentration or due to a lower cross-link density and therefore increase polymer chain mobility between cross-links. The softest materials were Factor II and Prestige and hence these materials may have the lowest overall cross-link density and filler concentration. A lower cross-link density indicates a greater distance between crosslinks (MC) allows the polymer chains between the cross-links to deform to greater distances producing a softer material. The end-linking of PDMS chains in Factor II also increases the distance between the cross-links and reduces the softness of the material.
A lower filler concentration reduces the polymer/ filler interactions and also increases the mobility of the polymer chains and helps to reduce the softness of the material. Prestige had a very low hardness but also very poor tensile strength and tear strength. This may be due to the presence of nonreinforcing additives such as non cross-linked polymer chains, plasticiser or non-reinforcing fillers present in the polymer base. These non-reinforcing additives reduce polymer/filler interactions and increase the mobility of the polymer chains reducing the base viscosity and the hardness of material. These additives effectively reduce the overall cross-link density and the tensile strength of the cross-linked network.

All the commercial materials tested had extremely high advancing contact angle measurements (90.8-114 $\left.\theta_{\text {Add }}\right)$ showing that these materials are not easily wetted by water. Poor surface wettability by saliva/water results in poor boundary lubrication (high surface friction) and increases patient discomfort. ${ }^{[8]}$ The high contact angles are due to the PDMS surface having a very low surface energy and thus preventing water from spreading over the surface. A new maxillofacial material would need a lower advancing contact angle and this may be achieved by the addition of silicone surfactants directly into the polymer matrix or by grafting hydrophilic surfactants onto the surface after activating the polymer surface by using argon plasma treatment.

The water absorption studies on the commercial materials allows us to determine which of these materials contain surface treated fillers. Cosmesil St and Prestige absorbed the largest amount of water after 12 months in distilled water at $37^{\circ} \mathrm{C}$. This may be due to the presence of hydrophilic nonsurface treated silica fillers present in the polymer matrix. The presence of - $\mathrm{OH}$ groups on the surface of the silica fillers helps to absorb water into the polymer matrix. ${ }^{[5]}$ Cosmesil HC, Factor II and Nusil had a negligible water absorption after 12 months in distilled water. This indicates the presence of surface treated hydrophobic silica fillers present in the polymer matrix. The surface groups on these silica particles repel water molecules and hence prevent water absorption into the material. ${ }^{[5]}$

In summary each of the materials tested had distinct advantages and disadvantages with regard their use as maxillofacial materials. Cosmesil HC and Nusil (MED-4920) had adequate mechanical properties in addition to good translucency which enables easier colour matching to patients skin. The high viscosity of these materials however made handling and mixing difficult and Cosmesil $\mathrm{HC}$ also was the hardest and therefore less 'skin like' 
material. Prestige (Premium facial and body elastomer) was a soft material however together with Cosmesil St it had extremely high water absorption and low tear strength. Factor II (A 2186) had good all round mechanical properties however it had the poorest wettability which may lead to patient discomfort when the prosthesis is in contact with skin. It is clear from this study that none of the commercially available materials tested possess all the ideal properties required to be a successful maxillofacial prosthetic material. There is therefore a clear need for an improved silicone rubber material specifically formulated for this unique medical use.

\section{References}

1 Chalian VA, Philips RW. Materials in maxillofacial prosthetics. Journal of Biomedical Materials Research Symposium 1974; 5:349-63.

2 Conroy B, Haylock C, Hulterstrom AK, Pratt G, Winter RW. Report of a four year research and development programme involving the Institute of Maxillofacial Technology and the University of Wales Institute of Science and Technology aimed at the production of a new facial prosthetic system. Proceedings Institute of Maxillofacial Technology 1979; 218-45.

3 Roberts AC. Silicones for facial prostheses. Dental Practitioner 1971;21:267-84.

4 Wolfaardt JF, Chandler HD, Smith BA. Mechanical properties of a new facial prosthetic material. Journal of Prosthetic Dentistry 1985;53:228-34.
5 Waters MGJ, Jagger RG, Winter RW. Effect of surfacemodified fillers on the water-absorption of a (RTV) silicone denture soft lining material. Journal of Dentistry 1996;24: 297-300.

6 Polyzois GL, Winter RW, Stafford GD. Boundary lubrication and maxillofacial prosthetic polydimethylsiloxanes. Biomaterials 1991;12:79-82.

7 Waters MGJ, Jagger RG, Polyzois GL. Wettability of silicone rubber maxillofacial prosthetic materials. Journal of Prosthetic Dentistry 1999;81:439-43.

8 Veres EM, Wolfaardt JF, Becker PJ. An evaluation of the surface characteristics of a facial prosthetic elastomer. Part I: Review of the literature on the surface characteristics of dental materials with maxillofacial prosthetic application. Journal of Prosthetic Dentistry 1990;63:193-7.

9 Veres EM, Wolfaardt JF, Becker PJ. An evaluation of the surface characteristics of a facial prosthetic elastomer. Part II: the surface texture. Journal of Prosthetic Dentistry 1990; 63:325-31.

10 Veres EM, Wolfaardt JF, Becker PJ. An evaluation of the surface characteristics of a facial prosthetic elastomer. Part III: wettability and hardness. Journal of Prosthetic Dentistry 1990;63:466-71.

11 Moore DJ, Glaser ZR, Tabacco MJ, Linebaugh MG. Evaluation of polymeric materials for maxillofacial prosthetics. Journal of Prosthetic Dentistry 1977;38:319-26.

12 Bell WT, Chalian VA, Moore BK. Poly(dimethylsiloxane) materials in maxillofacial prosthetics: Evaluation and comparison of physical properties. The Journal of Prosthetic Dentistry 1985;54:404-10.

13 Polyzois GL. Mechanical properties of 2 new additionvulcanising silicone prosthetic elastomers. International Journal of Prosthodontics 1999;12:359-62.

14 Shah GB, Winter RW. Effect of bimodality on tear properties of silicone networks. Macromolecular Chemistry and Physics 1996;197:2201-8. 